\title{
A ONE-STEP METHOD FOR THE NUMERICAL SOLUTION OF ORDINARY NON-LINEAR SECOND-ORDER DIFFERENTIAL EQUATIONS BASED UPON LOBATTO FOUR-POINT QUADRATURE FORMULA
}

\author{
K. D. SHARMA and R. G. GUPTA
}

(Received 24 July 1970; revised 4 August 1971)

Communicated by B. Mond

\begin{abstract}
This paper describes a one-step method based upon the Lobatto four-point quadrature formula for the numerical integration of differential equation: $y^{\prime \prime}(x)=f(x, y(x)$, $\left.y^{\prime}(x)\right) ; y\left(x_{0}\right)=y_{0}, y^{\prime}\left(x_{0}\right)=y_{0}^{\prime}$. The method has a local truncation error $0\left(h^{6}\right)$ in $y(x)$ and $0\left(h^{5}\right)$ in $y^{\prime}(x)$. In the case of linear second-order differential equation, a stability criterion has been developed. Theoretical and computational comparisons of the new method with existing method is discussed.
\end{abstract}

\section{Introduction}

The necessity of accurate numerical approximations to the solution of nonlinear differential equations governing physical systems has always been an important problem with scientists and engineers. Hammer and Hollingsworth [5] have used Gaussian quadrature methods for solving ordinary differential equations. This method has been further developed by Morrison and Stoller [10], Korganoff [9], Kuntzmann [8], Henrici [6], Day [2, 3], Jain and Sharma [7], Sharma [13]. Day [1] used this method for the solution of non-linear secondorder differential equations (first derivative absent) using Lobatto four-point quadrature formula.

The purpose of this paper is to investigate the use of Lobatto's four-point quadrature formula for solving the second-order non-linear differential equation: $y^{\prime \prime}(x)=f\left(x, y(x), y^{\prime}(x)\right) ; y\left(x_{0}\right)=y_{0}, y^{\prime}\left(x_{0}\right)=y_{0}^{\prime}$. The method has local truncation error $0\left(h^{6}\right)$ in $y(x)$ and $0\left(h^{5}\right)$ in $y^{\prime}(x)$. For $n$ integration steps the present method requires $(5 n+1)$ evaluations of $f$ as compared to the classical methods of Runge-Kutta, Runge-Kutta-Nyström's $4 n$. A stability criterion has been developed for the linear second-order differential equation. 


\section{Second-order differential equations}

Consider the second-order differential equation:

$$
y^{\prime \prime}(x)=f\left(x, y(x), y^{\prime}(x)\right) ; y\left(x_{0}\right)=y_{0}, y^{\prime}\left(x_{0}\right)=y_{0}^{\prime}
$$

where $x_{0}$ is an initial-point.

Throughout our discussion we shall assume that the function $f\left(x, y(x), y^{\prime}(x)\right)$ occurring in (2.1) is sufficiently differentiable to ensure that the derivations we give are valid on any context in which they are used.

Integrating (2.1) from $x_{0}$ to $x_{0}+h(h>0)$, we obtain the system of integral equations:

$$
\begin{aligned}
& y\left(x_{0}+h\right)=y_{0}+h y_{0}^{\prime}+\int_{x_{0}}^{x_{0}+h}\left[x_{0}+h-\tau\right] f\left(\tau, y(\tau), y^{\prime}(\tau)\right) d \tau \\
& y^{\prime}\left(x_{0}+h\right)=y_{0}^{\prime}+\int_{x_{0}}^{x_{0}+h} f\left(\tau, y(\tau), y^{\prime}(\tau)\right) d \tau
\end{aligned}
$$

We shall approximate the above two integrals by the Lobatto four-point quadrature formula on the interval $\left[x_{0}, x_{0}+h\right]$, see Hildebrand [4].

$$
\int_{x_{0}}^{x_{0}+h} F(x) d x=\frac{h}{2} \sum_{p=1}^{4} F\left(\tau_{p}\right) W_{p}+E_{4}
$$

where,

and

$$
\begin{array}{ll}
W_{1}=W_{4}=\frac{1}{6}, & W_{2}=W_{3}=\frac{5}{6}, \\
\tau_{1}=x_{0}, & \\
\tau_{2}=x_{0}+r h, & r=(5-\sqrt{ } 5) / 10 \\
\tau_{3}=x_{0}+s h, & s=(5+\sqrt{ } 5) / 10 \\
\tau_{4}=x_{0}+h &
\end{array}
$$

$$
E_{4}=-\frac{4 h^{7} F^{(6)}(\xi)}{3.2^{7} .15750}, \quad x_{0}<\xi<x_{0}+h
$$

We introduce the notations: $x_{r / 2}=x_{0}+r h / 2, y_{r / 2}=y\left(x_{r / 2}\right), x_{r}=x_{0}+r h$, $y_{r}=y\left(x_{r}\right) \cdots \cdots \cdots \ldots$. Then integral equations (2.3) and (2.4) can be replaced as:

$$
\begin{aligned}
y\left(x_{0}+h\right)= & y_{0}+h y_{0}^{\prime}+\left(h^{2} / 12\right)\left[f\left(x_{0}, y_{0}, y_{0}^{\prime}\right)+5\left\{r f\left(x_{s}, y_{s}, y_{s}^{\prime}\right)\right.\right. \\
& \left.\left.+s f\left(x_{r}, y_{r}, y_{r}^{\prime}\right)\right\}\right]+E
\end{aligned}
$$




$$
\begin{aligned}
y^{\prime}\left(x_{0}+h\right)= & y_{0}^{\prime}+(h / 12)\left[f\left(x_{0}, y_{0}, y_{0}^{\prime}\right)+f\left(x_{0}+h, y\left(x_{0}+h\right), y^{\prime}\left(x_{0}+h\right)\right)\right. \\
& \left.+5\left\{f\left(x_{r}, y_{r}, y_{r}^{\prime}\right)+f\left(x_{s}, y_{s}, y_{s}^{\prime}\right)\right\}\right]+E^{\prime}
\end{aligned}
$$

wh ere $E$ and $E^{\prime}$ are truncation errors of $0\left(h^{6}\right)$ and $O\left(h^{5}\right)$ respectively.

We note that we do not know $y_{r}, y_{s}, y_{r}^{\prime}, y_{s}^{\prime}$; thus if such an algorithm is to be of computational value, we must obtain accurate approximate values for $y_{r}, y_{s}$, $y_{r}^{\prime}$ and $y_{s}^{\prime}$. For this, we obtain the following Hermite expressions for $y_{r}$ and $y_{r}^{\prime}$ (we shall denote $\sqrt{5}$ by $p$ ):

$$
\begin{gathered}
y_{r}=y_{0}+r h y_{0}^{\prime}+\frac{(r h)^{2}}{6}\left[y^{\prime \prime}+2 f\left(x_{r / 2}, y_{r / 2}, y_{r / 2}^{\prime}\right)\right]+E_{r} \\
y_{r}^{\prime}=\frac{(25-19 p)}{2 h} y_{0}+(3-2 p) y_{0}^{\prime}+\frac{h(5-3 p)}{20} y_{0}^{\prime \prime}+\frac{(15+p)}{2 h} y_{r} \\
+\frac{(9 p-20)}{h} y_{s}+E_{r}^{\prime}
\end{gathered}
$$

where $E_{\mathrm{r}}$ and $E_{r}^{\prime}$ are truncation errors of $O\left(h^{5}\right)$ and $O\left(h^{4}\right)$ respectively. Similar expressions for $y_{s}$ and $y_{s}{ }^{\prime}$ can be obtained by changing $r$ to $s$ and $p$ to $-p$ in equations (2.7) and (2.8) respectively.

The approximate values of $y_{r / 2}^{\prime}$ occurring in the equation (2.7) can be obtained by halving the values of $r, s$ and $h$ in (2.8) and the approximate values of $y_{r / 2}$ can be obtained by Taylor's theorem in conjunction with (2.1). Substituting the values of $y_{r / 2}$ and $y_{r / 2}^{\prime}$ in (2.7) we obtain the approximate values of $y_{r}$ to the desired order of accuracy. Similar calculations yield the values of $y_{s}, y_{r}^{\prime}$ and $y_{s}^{\prime}$ Finally, these values are used in (2.5) to obtain the desired value of $y\left(x_{0}+h\right)$. To calculate $y^{\prime}\left(x_{0}+h\right)$ we consider the expression:

$$
\begin{gathered}
y^{\prime}\left(x_{0}+h\right)=-\frac{33}{h} y_{0}-7 y_{0}^{\prime}-\frac{h}{2} y_{0}^{\prime \prime}+\frac{25(1+p)}{2 h} y_{r}+\frac{25(1-p)}{2 h} y_{s} \\
+\frac{8}{h} y\left(x_{0}+h\right)+\frac{h^{5}}{150} f^{\prime \prime \prime}\left(x_{0}, y_{0}, y_{0}^{\prime}\right)+0\left(h^{6}\right)
\end{gathered}
$$

Substitution of the values of $f\left(x_{r}, y_{r}, y_{r}^{\prime}\right), f\left(x_{s}, y_{s}, y_{s}^{\prime}\right)$ calculated above along with the newly calculated value of $\left.f\left(x_{0}+h\right), y\left(x_{0}+h\right), y^{\prime}\left(x_{0}+h\right)\right)$ into the equation (2.6) leads to the required value of $y^{\prime}\left(x_{0}+h\right)$.

\section{Error Terms}

Error terms for each of the expressions (as abbreviated above by $E_{r}, E_{r}^{\prime}, E, E^{\prime}$ ) have been calculated as follows:

$$
\begin{aligned}
E_{r}= & h^{5}\left[-\frac{r^{5}}{720} f^{\prime \prime \prime}\left(x_{0}, y_{0}, y_{0}^{\prime}\right)+\frac{r^{2}}{2880}(26-10 p) \tau_{r}+(4 p+8) \tau_{s}\right] \\
& \left.\times f^{\prime \prime}\left(x_{0}, y_{0}, y_{0}^{\prime}\right) f_{y^{\prime}}^{\prime}\left(x_{0}, y_{0}, y_{0}^{\prime}\right)-\frac{r^{5}}{2880} f^{I V}\left(x_{0}, y_{0}, y_{0}^{\prime}\right)\right]+0\left(h^{6}\right)
\end{aligned}
$$


where

$$
\begin{gathered}
\tau_{r}=\frac{\xi_{r}-x_{0}}{h}, \quad \tau_{s}=\frac{\xi_{s}-x_{0}}{h} \\
E_{r}^{\prime}=h^{4}\left[\frac{(3-p)}{2,400} f^{\prime \prime}\left(x_{0}, y_{0}, y_{0}^{\prime}\right)+\left\{\frac{(15+p)}{2} R_{1}+(9 p-20) R_{2}\right\}\right]+0\left(h^{5}\right) \\
E=\frac{5 h^{6}}{12}\left[r\left\{\frac{(44+25 p)}{3600} f^{\prime \prime \prime}\left(x_{0}, y_{0}, y_{0}^{\prime}\right)+R_{1}\right\}+s\left\{\frac{(44-25 p)}{3600}\right.\right. \\
\left.\left.f^{\prime \prime \prime}\left(x_{0}, y_{0}, y_{0}^{\prime}\right)+R_{2}\right\}\right] \times f_{y^{\prime}}\left(x_{0}, y_{0}, y_{0}^{\prime}\right)+0\left(h^{7}\right)
\end{gathered}
$$

and

$$
E^{\prime}=\frac{h^{5}}{12}\left[\frac{11}{90} f^{\prime \prime \prime}\left(x_{0}, y_{0}, y_{0}^{\prime}\right)+5\left(R_{1}+R_{2}\right)\right] f_{y^{\prime}}\left(x_{0}, y_{0}, y_{0}^{\prime}\right)+0\left(h^{6}\right)
$$

where $R_{1}$ and $R_{2}$ are the coefficients of $h^{5}$ in $E_{r}$ and $E_{s}$ respectively.

\section{Stability criterion}

The stability of the present method will be discussed in a manner similar to that adopted by Jain and Sharma [7]. Consider the differential equation $y^{\prime \prime}=\alpha y$; $\alpha$ is a real number. We shall discuss the three cases $\alpha=0,-k^{2},+k^{2}$ respectively. If we insert the values of $y_{n+r}, y_{n+s}, y_{n+r}^{\prime}, y_{n+s}^{\prime}$ in $y_{n+1}$ and $y_{n+1}$ in the algorithm, we obtain

$$
\left[\begin{array}{l}
y_{n+1} \\
y_{n+1}^{\prime}
\end{array}\right]=\left[\begin{array}{ll}
1+\frac{h^{2} \alpha}{2}+\frac{h^{4} \alpha^{2}}{24}+\frac{h^{6} \alpha^{3}}{720} & h+\frac{h^{3} \alpha}{6}+\frac{h^{5} \alpha^{2}}{120} \\
h+\frac{h^{3} \alpha^{2}}{6}+\frac{h^{5} \alpha^{3}}{120}+\frac{h^{7} \alpha^{4}}{8640} & 1+\frac{h^{2} \alpha}{2}+\frac{h^{4} \alpha^{2}}{24}+\frac{h^{6} \alpha^{3}}{1440}
\end{array}\right]\left[\begin{array}{l}
y_{n} \\
y_{n}^{\prime}
\end{array}\right]
$$

For $\alpha=0$, we have: $y_{n+1}=y_{n}+h y_{n}^{\prime}$ and $y_{n+1}^{\prime}=y_{n}^{\prime}$. The solution of this system can be written as $y_{n}^{\prime}=y_{0}^{\prime}$ and $y_{n}=y_{0}+n h y_{0}^{\prime}$, which is an expected result.

We now consider the case $\alpha=-k^{2}$; the solution in this case is oscillating. We therefore consider the eigenvalues of the matrix in (3.1). We find that eigenvalues have unit modulus for $0 \leqq h^{2} k^{2} \leqq 6.8$. The deviation of the modulus of the eigenvalues from unity is less than 0.01 for $6.8<h^{2} k^{2} \leqq 9.5$. This shows that the method is stable for a sufficiently wide range of the values of $h^{2} k^{2}$. The same has also been observed in the case of sample examples computed for the method. The Runge-Kutta method as studied for the above problem $y^{\prime \prime}=-k^{2} y$ is stable for $0 \leqq h^{2} k^{2} \leqq 7.756$, and the range of stability for the Runge-KuttaNyström method for the same equation is $0 \leqq h^{2} k^{2} \leqq 6.690$. 
For the case $y^{\prime \prime}=k^{2} y$, of which the solutiors are exponential in nature, we rely on the analysis given by Rutishauser $[11,12]$. It is well known that the solution of the differential equation $y^{\prime \prime}=k^{2} y$, written in the matrix form is:

$$
\left[\begin{array}{l}
y(x) \\
y^{\prime}(x)
\end{array}\right]=\exp \left[\left(x-x_{0}\right) M\right]\left[\begin{array}{l}
y_{0} \\
y_{0}^{\prime}
\end{array}\right] \text {, where } M=\left[\begin{array}{cc}
0 & 1 \\
k^{2} & 0
\end{array}\right]
$$

For the point $x=x_{0}+n h$, this solution can be written in the form (with $\alpha=k^{2}$ ):

$$
\left[\begin{array}{l}
y(x) \\
y^{\prime}(x)
\end{array}\right]=\left[\begin{array}{ll}
a_{11} & a_{12} \\
a_{21} & a_{22}
\end{array}\right]\left[\begin{array}{l}
y_{0} \\
y_{0}^{\prime}
\end{array}\right]
$$

It may be observed that:

$$
\underset{h \rightarrow 0}{\operatorname{Lt}} \frac{1}{h^{6}}\left[\left\{\exp (M h)-\left[\begin{array}{ll}
a_{11} & a_{12} \\
a_{21} & a_{22}
\end{array}\right]\right\}\right]=\left[\begin{array}{rr}
0 & 0 \\
0 & +\frac{k^{6}}{1440}
\end{array}\right]
$$

where

$$
\begin{aligned}
& a_{11}=1+\frac{h^{2} k^{2}}{2}+\frac{h^{4} k^{4}}{24}+\frac{h^{6} k^{6}}{720}+\cdots \cdots \\
& a_{12}=h+\frac{h^{3} k^{2}}{6}+\frac{h^{5} k^{4}}{720}+\cdots \cdots \\
& a_{21}=h k^{2}+\frac{h^{3} k^{4}}{6}+\frac{h^{5} k^{6}}{120}+\frac{h^{7} k^{8}}{8640}+\cdots \cdots \\
& a_{22}=1+\frac{h^{2} k^{2}}{2}+\frac{h^{4} k^{4}}{24}+\frac{h^{6} k^{6}}{1440}+\cdots \cdots
\end{aligned}
$$

This indicates that the present method is of order five by definition of Rutishauser. In the case of a large number of integration intervals (large $x$, small $h$ ), the relative error of this method, for equation $y^{\prime \prime}=k^{2} y$ can be considered as follows:

The maximum eigen-value of the matrix in (3.1) for $\alpha=k^{2}$ is given by:

$$
\begin{aligned}
\lambda & =(1 / 2)\left[\left(a_{11}+a_{22}\right)+\sqrt{\left[\left(a_{11}-a_{22}\right)^{2}+4 a_{12} a_{21}\right]}\right] \\
& =1+h k+\frac{h^{2} k^{2}}{2}+\frac{h^{3} k^{3}}{6}+\frac{h^{4} k^{4}}{24}+\frac{h^{5} k^{5}}{720}-\frac{13 h^{7} k^{7}}{2880}
\end{aligned}
$$

and therefore the relative error $F$ (in the sense of Rutishauser) of the method is:

$$
F_{\infty} \simeq \frac{h k-\log \lambda}{h}
$$




$$
\begin{aligned}
& =\frac{\log \left(e^{h k}\right)-\log \lambda}{h} \\
& =\frac{1}{h} \log \left[1+\frac{\left(e^{h k}-\lambda\right)}{\lambda}\right] \\
& \simeq \frac{19 h^{6} k^{7}}{4032} \text { for large } x \text { and small } h .
\end{aligned}
$$

Rutishauser (1960) calculates the relative error for the Runge-Kutta-Nyström method for the equation $y^{\prime \prime}=k^{2} y$ and finds that $F_{\infty} \simeq\left(h^{4} k^{5} / 320\right)$; for the Runge-Kutta method he obtained $F_{\infty} \simeq h^{4} k^{5} / 120$.

\section{Algorithm}

To advance from $x_{n}$ to $x_{n+1}\left(x_{n+1}=x_{n}+h\right)$ we note that $f\left(x_{n}, y_{n}, y_{n}^{\prime}\right)$ has been computed in the previous step. The computation should proceed as follows:

Calculate,

$$
\begin{aligned}
y_{n+r / 2}= & y_{n}+\frac{r h}{2} y_{n}^{\prime}+\frac{r^{2} h^{2}}{8} y_{n}^{\prime \prime} \\
y_{n+s / 2}= & y_{n}+\frac{s h}{2} y_{n}^{\prime}+\frac{s^{2} h^{2}}{8} y_{n}^{\prime \prime} \\
y_{n+r / 2}^{\prime}= & \frac{(25-19 p)}{h} y_{n}+\frac{(3-2 p)}{1} y_{n}^{\prime}+\frac{(5-3 p) h}{40} y_{n}^{\prime \prime}+\frac{(15+p)}{h} y_{n+r / 2} \\
& \frac{(25+19 p)}{h} y_{n}+\frac{(3+2 p)}{1} y_{n}^{\prime}+\frac{(5+3 p) h}{40} y_{n}^{\prime \prime}+\frac{(15-p)}{h} y_{n+s / 2} \\
y_{n+s / 2}^{\prime}= & -\frac{2(9 p+20)}{h} y_{n+r / 2}
\end{aligned}
$$

Then,

$$
\begin{gathered}
y_{n+r}=y_{n}+r h y_{n}^{\prime}+\frac{(r h)^{2}}{6}\left[f\left(x_{n}, y_{n}, y_{n}^{\prime}\right)+2 f\left(x_{n+r / 2}, y_{n+r / 2}, y_{n+r / 2}^{\prime}\right)\right] \\
y_{n+s}=y_{n}+s h y_{n}^{\prime}+\frac{(s h)^{2}}{6}\left[f\left(x_{n}, y_{n}, y_{n}^{\prime}\right)+2 f\left(x_{n+s / 2}, y_{n+s / 2}, y_{n+s / 2}^{\prime}\right)\right] \\
y_{n+r}^{\prime}=\frac{(25-19 p)}{2 h} y_{n}+(3-2 p) y_{n}^{\prime}+\frac{(5-3 p) h}{20} y_{n}^{\prime \prime}+\frac{(15-p)}{2 h} y_{n+r} \\
+\frac{(9 p-20)}{h} y_{n+s}
\end{gathered}
$$




$$
\begin{aligned}
y_{n+s}^{\prime}=\frac{(25+19 p)}{2 h} y_{n}+(3+2 p) y_{n}^{\prime}+\frac{(5+3 p) h}{20} y_{n}^{\prime \prime} & \frac{(15+p)}{2 h} y_{n+s} \\
& -\frac{(9 p+20)}{h} y_{n+r}
\end{aligned}
$$

Finally,

$y_{n+1}=y_{n}+h y_{n}^{\prime}+\frac{h^{2}}{12}\left[f\left(x_{n}, y_{n}, y_{n}^{\prime}\right)+5\left\{s f\left(x_{n+r}, y_{n+r}, y_{n+r}^{\prime}\right)+r f\left(x_{n+s}, y_{n+s}, y_{n+s}^{\prime}\right)\right\}\right]$

Again calculate,

$$
y_{n+1}^{\prime}=-\frac{33}{h} y_{n}-7 y_{n}^{\prime}-\frac{h}{2} y_{n}^{\prime \prime}+\frac{25(1+p)}{2 h} y_{n+r}+\frac{25(1-p)}{2 h} y_{n+s}+\frac{8}{h} y_{n+1}
$$

Thus,

$$
\begin{aligned}
y_{n+1}^{\prime}=y_{n}^{\prime}+\frac{h}{12}\left[f\left(x_{n}, y_{n}, y_{n}^{\prime}\right)+5\left\{f\left(x_{n+r}, y_{n+r}, y_{n+r}^{\prime}\right)+\right.\right. & \left.f\left(x_{n+s}, y_{n+s}, y_{n+s}^{\prime}\right)\right\} \\
& \left.+f\left(x_{n+1}, y_{n+1}, y_{n+1}^{\prime}\right)\right]
\end{aligned}
$$

\section{Numerical results of the method}

For the purpose of computational comparison of this method with other methods, we consider the following two examples. We have written programs for the ICL 1909 Computer in FORTRAN IV (in single precision) for the method under consideration and the results have been compared with the exact results and the results obtained by other methods.

\section{Example One}

The differential equation

$$
\frac{d^{2} y}{d x^{2}}+\left(16 \pi^{2} e^{-2 x}-1 / 4\right) y=0
$$

with initial conditions:

has the solution:

$$
y(0)=1.0 ;\left(\frac{d y}{d x}\right)_{x=0}=0.5
$$

$$
y(x)=e^{x / 2} \cos \left(4 \pi e^{-x}\right) .
$$

We computed approximate values of $y(x)$ at equally spaced points with $h=0.02$ and obtained the approximate values listed in Table one. 
TABLE ONE

\begin{tabular}{rrrrr}
\hline $\boldsymbol{x}$ & Nyström & Runge-Kutta & New Method & \multicolumn{1}{c}{ Exact } \\
\hline & & & & \\
2.0 & -000.35227603 & -000.35227603 & -000.35205017 & -000.35205060 \\
4.0 & 007.19395636 & 007.19395636 & 007.19420981 & 007.19420412 \\
6.0 & 020.07545103 & 020.07545103 & 020.07580847 & 020.07579367 \\
8.0 & 054.59685889 & 054.59685888 & 054.59770481 & 054.59766497 \\
10.0 & 148.41099023 & 148.41099021 & 148.41324328 & 148.41313528 \\
\hline
\end{tabular}

\section{Example Two}

The Legendre differential equation :

$$
\left(1-x^{2}\right) \frac{d^{2} y}{d x^{2}}-2 x \frac{d y}{d x}+72 y=0
$$

with initial conditions:

has the solution:

$$
x=0, y(0)=0.2734375000,\left(\frac{d y}{d x}\right)_{x=0}=0
$$

$$
y(x)=\left(6435 x^{8}-12012 x^{6}+6930 x^{4}-1260 x^{2}+35\right) / 128 .
$$

We computed approximate values of $y(x)$ at equally spaced points with $h=0.02$ and obtained the approximate values listed in Table two.

TABLE TWO

\begin{tabular}{rrrrr}
\hline $\boldsymbol{x}$ & Nyström & Runge-Kutta & New Method & \multicolumn{1}{c}{ Exact } \\
\hline & & & & \\
0.10 & 0.1803210877 & 0.1803217228 & 0.1803207210 & 0.1803207215 \\
0.20 & -0.0395736736 & -0.0395615211 & -0.0395647992 & -0.0395548000 \\
0.30 & -0.2390734252 & -0.2390709065 & -0.2390745826 & 0.2390745910 \\
0.40 & -0.2669997410 & -0.2669999266 & -0.2669992858 & -0.2669993000 \\
0.50 & -0.0736421547 & -0.0736478032 & -0.0736388781 & -0.0736389159 \\
\hline
\end{tabular}

\section{Conclusion}

We observe that the one-step Lobatto method developed here compares favourably with other methods. The relative error of this method is relatively much smaller than the classical Runge-Kutta and Nyström methods with only $(n+1)$ additional functional evaluations for $n$ steps. 


\section{Acknowledgement}

We are thankful to the Council of Scientific and Industrial Research (India), for providing financial assistance during the preparation of this paper. We are grateful to Prof. R. N. Dogra, Prof. M. P. Singh and Prof. J. C. Shouri for providing us all the facilities in carrying out this project. We are indebted to the referee for his valuable suggestions for improving the presentation of this paper.

\section{References}

[1] J. T. Day, 'A Runge-Kutta method for the numerical integration of the differential equation $y^{\prime \prime}=f(x, y)^{\prime}, Z A M M 45$ (1965), 354-356.

[2] J. T. Day, 'A one-step method for the numerical solution of second-order linear differential equations', Math. Comp. 18 (1964), 664-68.

[3] J. T. Day, 'A one-step method for the numerical integration of the differential equation $y^{\text {' }=}$ $f(x) y+g(x)$, Computer Journal, 7 (1964), 314-17.

[4] F. B. Hildebrand, Introduction to Numerical Analysis (McGraw-Hill, New York (1956)).

[5] P. C. Hammer and J. W. Hollingsworth, 'Trapezoidal methods of approximating solution of differential equations' MTAC 9 (1955), 92-96.

[6] P. Henrici, Discrete variable methods in ordinary differential equations (John Wiley and Sons, Inc., New York (1962)).

[7] M. K. Jain and K. D. Sharma, 'Numerical solution of linear differential equations and Volterra's integral equation using Lobatto quadrature formula', Computer Journal, 10 (1967) 101-106.

[8] J. Kuntzmann, 'Neuere Entwicklungen der Method von Runge and Kutta', $Z A M M, 41$ (1961) T 28-T 31.

[9] A. Korganoff, 'Sur les formulas d'intégration numérique des equations différentielles donnant une approximation dordre élevé', Chiffers 1 (1958), 171-180.

[10] D. Morrison and L. Stoller, 'A method for numerical integration of ordinary differential equations', MTAC 12 (1958), 269-272.

[11] H. Rutishauser, 'Ueber die Instabilitäte von Methoden zur Integration gewöhnlicher Differentialgleichugen', $Z A M P 3$ (1952) 65-74.

[12] H. Rutishauser, 'Bemerkungen numerischen Integration gewöhnlicher Differentialgleichungen $n$-ter ordnung', Num. Math. 2 (1960) 263-79.

[13] K. D. Sharma, 'One-step methods for the numerical solution of linear differential equations based upon Lobatto quadrature formulae,' Jour. Austr. Math. Soc. 11 (1970), 115-128.

\section{Computer Centre}

Indian Institute of Technology

and

Department of Mathematics*

Indian Institute of Technology

Hauz Khas, New Delhi-29

India

* Present address:

Computer Center

Indian Institute of Technology 